\title{
Total alkaloids of Rubus alceifolius Poir inhibit tumor angiogenesis through suppression of the Notch signaling pathway in a mouse model of hepatocellular carcinoma
}

\author{
JINYAN ZHAO ${ }^{1,2}$, WEI LIN ${ }^{1,2}$, ZHIYUN CAO $^{1}$, QUNCHUAN ZHUANG ${ }^{1,2}$, \\ LIANGPU ZHENG ${ }^{1,2}$, JUN PENG $^{1,2}$ and ZHENFENG HONG ${ }^{1,2}$ \\ ${ }^{1}$ Academy of Integrative Medicine; ${ }^{2}$ Fujian Key Laboratory of Integrative Medicine on Geriatrics, \\ Fujian University of Traditional Chinese Medicine, Fuzhou, Fujian 350122, P.R. China
}

Received October 21, 2013; Accepted May 2, 2014

DOI: $10.3892 / \mathrm{mmr} .2014 .2702$

\begin{abstract}
Angiogenesis, which has a critical role in human tumor growth and development, is tightly regulated by the Notch signaling pathway. Total alkaloids are active components of the plant Rubus alceifolius Poir, which is used for the treatment of various types of cancer. A previous study by our group showed that the total alkaloids of Rubus alceifolius Poir (TARAP) induced hepatocellular carcinoma (HCC) cell apoptosis through the activation of the mitochondria-dependent pathway in vitro and in vivo, as well as inhibited angiogenesis in a chick embryo chorioallantoic membrane model. In the present study, to further analyze the specific mechanisms underlying the antitumor activity of TARAP, a HCC xenograft mouse model was used to assess the effect of TARAP on angiogenesis in vivo. TARAP was found to suppress the expression of vascular endothelial growth factor (VEGF) A and VEGF receptor-2 in tumor tissues, which resulted in the inhibition of tumor angiogenesis. In addition, TARAP treatment was observed to inhibit the expression of Notch1, delta-like ligand 4 and jagged 1, which are key mediators of the Notch signaling pathway. The present study identified that the inhibition of tumor angiogenesis through the suppression of the Notch signaling pathway may be one of the mechanisms through which TARAP may be effective in the treatment of cancer.
\end{abstract}

\footnotetext{
Correspondence to: Professor Zhenfeng Hong or Dr Jun Peng, Academy of Integrative Medicine, Fujian University of Traditional Chinese Medicine, 1 Huatuo Road, Fuzhou, Fujian 350122, P.R. China E-mail: zfhong1953@163.com

E-mail: pjunlab@hotmail.com
}

Key words: total alkaloids of Rubus alceifolius Poir, hepatocellular carcinoma, tumor angiogenesis, Notch signaling pathway

\section{Introduction}

Hepatocellular carcinoma (HCC) is the third most common cause of cancer-associated mortality worldwide. The HCC incidence rate is increasing more rapidly than most other types of cancers due to the increasing prevalence of hepatitis B and C viral infections $(1,2)$. Therapies for HCC include surgery, radiofrequency ablation and chemotherapy. At present, no drugs are available to prevent or reduce tumor spread and/or recurrence, which severely affects prognosis and survival. HCC is the only type of cancer in which 'conventional' chemotherapeutics must be avoided, due to the underlying liver damage. Thus, a novel drug for the treatment of HCC is urgently required.

Tumor angiogenesis is a complex process in which new blood vessels are formed in response to interactions between tumor cells and endothelial cells (ECs) $(3,4)$. Angiogenesis has an important role in cancer growth and metastasis, with tumors able to grow autonomously to a size of $2 \pm 3 \mathrm{~mm}^{3}$, but incapable of growing beyond this size without a blood supply (5-9). Tumor cells continuously stimulate the formation of new blood vessels to grow. New blood vessels that surround tumor tissues provide a gateway for the tumor cells to enter the vascular system and metastasize to distant organs. Thus, the inhibition of angiogenesis has been proposed to suppress tumor growth and metastasis (10). An increasing number of studies have revealed that tumor cells secrete angiogenic growth factors to stimulate EC proliferation and to induce angiogenesis (6). Among them, vascular endothelial growth factor (VEGF) is one of the most potent angiogenic factors and it is overexpressed in numerous types of human cancer (11).

Genetic studies have shown that Notch signaling has a critical role in vascular formation during early embryonic development (12-14). Notch signaling is an evolutionarily conserved pathway, which regulates cell fate decisions during the embryonic development of vertebrates and invertebrates (15). There are four mammalian Notch genes, Notch1-4, and five ligands, jagged 1, jagged 2, delta-like ligand (DLL) 1, DLL3 and DLL4 $(12,16,17)$. Notch signaling has been reported to have a crucial role in tumor angiogenesis 
and is a novel target for anti-angiogenic therapy. Studies have demonstrated that the activation of the Notch signaling pathway inhibits angiogenesis. Activated Notch1 and Notch4 have been reported to decrease the expression of VEGF receptor (VEGFR) 2, resulting in decreased human EC proliferation in response to VEGF (18). Human ECs transduced with DLL4 have been shown to exhibit decreased VEGF-induced proliferation and migration, as well as a significant reduction in the expression of VEGFR2 and its coreceptor neuropilin-1, suggesting that DLL4 is an inhibitor of angiogenesis (19). In addition, inhibiting jagged using an antisense oligomer in bovine ECs has been found to induce tube formation and collagen gel invasion in response to fibroblast growth factor, but not VEGF (20). Although Notch signaling has been reported to inhibit sprouting angiogenesis in these studies (18-20), most were performed in vitro. Thus, in vivo tumor angiogenesis assays are required to confirm these findings and to determine their physiological relevance.

Rubus alceifolius Poir is a natural product, which is used in Traditional Chinese Medicine in Southern regions of Fujian Province, China, to treat various types of hepatitis. In a previous study, our group identified that total alkaloids of Rubus alceifolius Poir (TARAP) promoted apoptosis in human HCC cells (21). However, the specific mechanism underlying the anticancer effect of TARAP in vivo has yet to be elucidated. In the present study, in order to further investigate the anticancer activity of TARAP, the effect of TARAP on tumor growth and its underlying molecular mechanisms were investigated in a HCC xenograft mouse model.

\section{Materials and methods}

Materials and reagents. Dulbecco's modified Eagle's medium (DMEM), fetal bovine serum (FBS), penicillin-streptomycin, Trypsin-EDTA and TRIzol ${ }^{\circledR}$ reagent were purchased from Life Technologies (Carlsbad, CA, USA). SuperScript ${ }^{\circledR}$ II reverse transcriptase was purchased from Promega Corporation (Madison, WI, USA). Antibodies against cluster of differentiation (CD) 31 (human, pAb), VEGF-A (human, pAb), VEGFR2 (human, pAb), Notch (human, mAb), Dll4 (human, pAb) and jagged 1 (human, pAb) were obtained from Santa Cruz Biotechnology, Inc. (Santa Cruz, CA, USA). All antibodies were diluted 1:100. All other chemicals, unless otherwise stated, were purchased from Sigma-Aldrich (St. Louis, MO, USA).

TARAP preparation. TARAP preparation was performed as described previously (21). The roots of Rubus alceifolius Poir were collected in Anxi (China) and were identified and authenticated at Fujian University of Traditional Chinese Medicine (Fuzhou, China). The alkaloids were then extracted as follows: The herb powder (1 g) was extracted using $50 \mathrm{ml}$ chloroform:methanol:ammonia solution $(15: 4: 3)$ for $2 \mathrm{~h}$ in an ice bath, sonicated for $30 \mathrm{~min}$, brought to room temperature and filtered. The filtered solution was collected and dessicated. The resultant residue was dissolved in $2 \mathrm{ml}$ of $2 \%$ sulfuric acid solution and filtered. The filter paper and residue were re-washed with $2 \mathrm{ml}$ of $2 \%$ sulfuric acid solution and buffer solution ( $\mathrm{pH}$ 3.6). The buffer was then added to make a final volume of $50 \mathrm{ml}$, and the solution was saved for future use. Acid dye colorimetry was used to measure the total alkaloid content. The total alkaloid content was $0.81 \mathrm{mg}$ alkaloid per gram of initial herb powder.

Cell culture and animals. HepG2 human HCC cells were purchased from the American Type Culture Collection (Rockville, MD, USA). Cells were grown in DMEM containing $10 \%$ (v/v) FBS, $100 \mathrm{U} / \mathrm{ml}$ penicillin and $100 \mu \mathrm{g} / \mathrm{ml}$ streptomycin in a humidified incubator at $37^{\circ} \mathrm{C}$ with $5 \% \mathrm{CO}_{2}$. Cells were subcultured to $80-90 \%$ confluency. Six-week-old male BALB/c athymic nude mice were purchased from Shanghai Si-Lai-Ke Experimental Animal Ltd. (Shanghai, China). Mice were housed at five mice per cage in a pathogen-free room in an environment with controlled temperature $\left(22^{\circ} \mathrm{C}\right)$, humidity and a 12-h light/dark cycle with free access to water and a standard laboratory diet. All animal treatments were performed in accordance with the Guidelines for Animal Experimentation of Fujian University of Traditional Chinese Medicine (Fuzhou, China). The animal studies were approved by the Fujian Institute of Traditional Chinese Medicine Animal Ethics Committee (Fuzhou, China).

In vivo tumor xenograft study. HepG2 cells were seeded at a density of $4 \times 10^{6}$ cells mixed 1:1 with Matrigel ${ }^{\mathrm{TM}}$ and were subcutaneously injected in the right flank of the mice to initiate tumor growth. Seven days following xenograft implantation, when the tumors reached $3 \mathrm{~mm}$ in diameter, the mice were randomly divided into two groups $(n=10)$ and were intragastrically administered $3 \mathrm{~g} / \mathrm{kg}$ body weight TARAP or saline daily, five days a week for 21 days. At the end of the experiment, the animals were anaesthetized with ether and the mice were sacrificed by cervical vertebra dislocation, and the tumor tissue was removed. A section of the tumor was fixed in buffered formalin and the remaining sections were stored at $-80^{\circ} \mathrm{C}$ for molecular analyses.

RNA extraction and reverse transcription-polymerase chain reaction $(P C R)$ analysis. Total RNA was isolated using TRIzol Reagent. Oligo(dT)-primed RNA $(1 \mu \mathrm{g})$ was reverse-transcribed using SuperScript II reverse transcriptase according to the manufacturer's instructions. The cDNA product $(1 \mu \mathrm{l})$ was added to prepare the PCR reaction mixture containing $200 \mu \mathrm{M}$ dNTPs, $1.5 \mathrm{mM} \mathrm{MgCl}_{2}$ and $1.25 \mathrm{U}$ Platinum Taq polymerase for VEGF-A, VEGFR2, Notch, Dll4 or jagged1 with a Taq DNA polymerase. GAPDH was used as an internal control. Samples were analyzed using gel electrophoresis on a $1.5 \%$ agarose gel. DNA bands were analyzed using a Bio-Rad Gel Doc 2000 System (Bio-Rad, Hercules, CA, USA).

Immunohistochemistry (IHC). Tumor samples were fixed with $10 \%$ formaldehyde for $12 \mathrm{~h}$ and processed for the generation of paraffin-embedded tumor slides. Slides were subjected to antigen retrieval and endogenous peroxidase activity was blocked using hydrogen peroxide. Sections were then incubated with CD31, VEGF-A, VEGFR2, Notch, Dll4 and jagged 1 antibodies diluted 1:100. Subsequent to washing with phosphate-buffered saline (PBS), slides were incubated with biotinylated secondary antibodies followed by incubation with conjugated horseradish peroxidase-labeled streptavidin (Dako UK Ltd., Ely, UK) and washed with PBS. Slides were then incubated with diaminobenzidine as the chromogen, followed by counterstaining with 

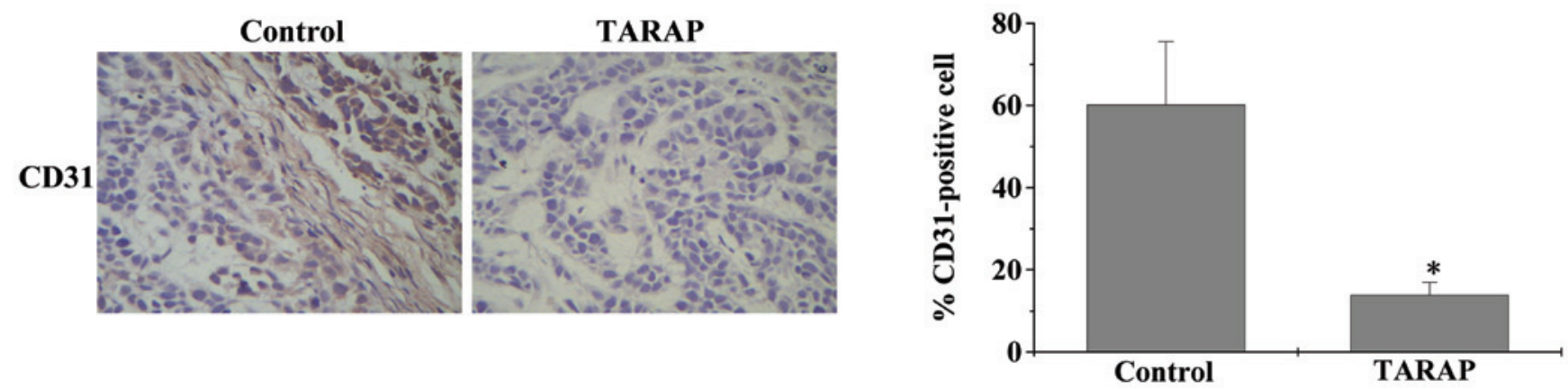

Figure 1. Effect of TARAP on intratumoral microvessel density in HCC xenograft mice. Tumor tissues were processed for immunohistochemical staining for CD31. Images were captured at a magnification of x400. Immunohistochemical staining was quantified and presented as a percentage of positively-stained cells. Data are presented as the mean \pm standard deviation from 10 individual mice in each group. "P $<0.05$ vs. control group. TARAP, total alkaloids of Rubus alceifolius Poir; HCC, hepatocellular carcinoma; CD, cluster of differentiation.

$\mathbf{A}$
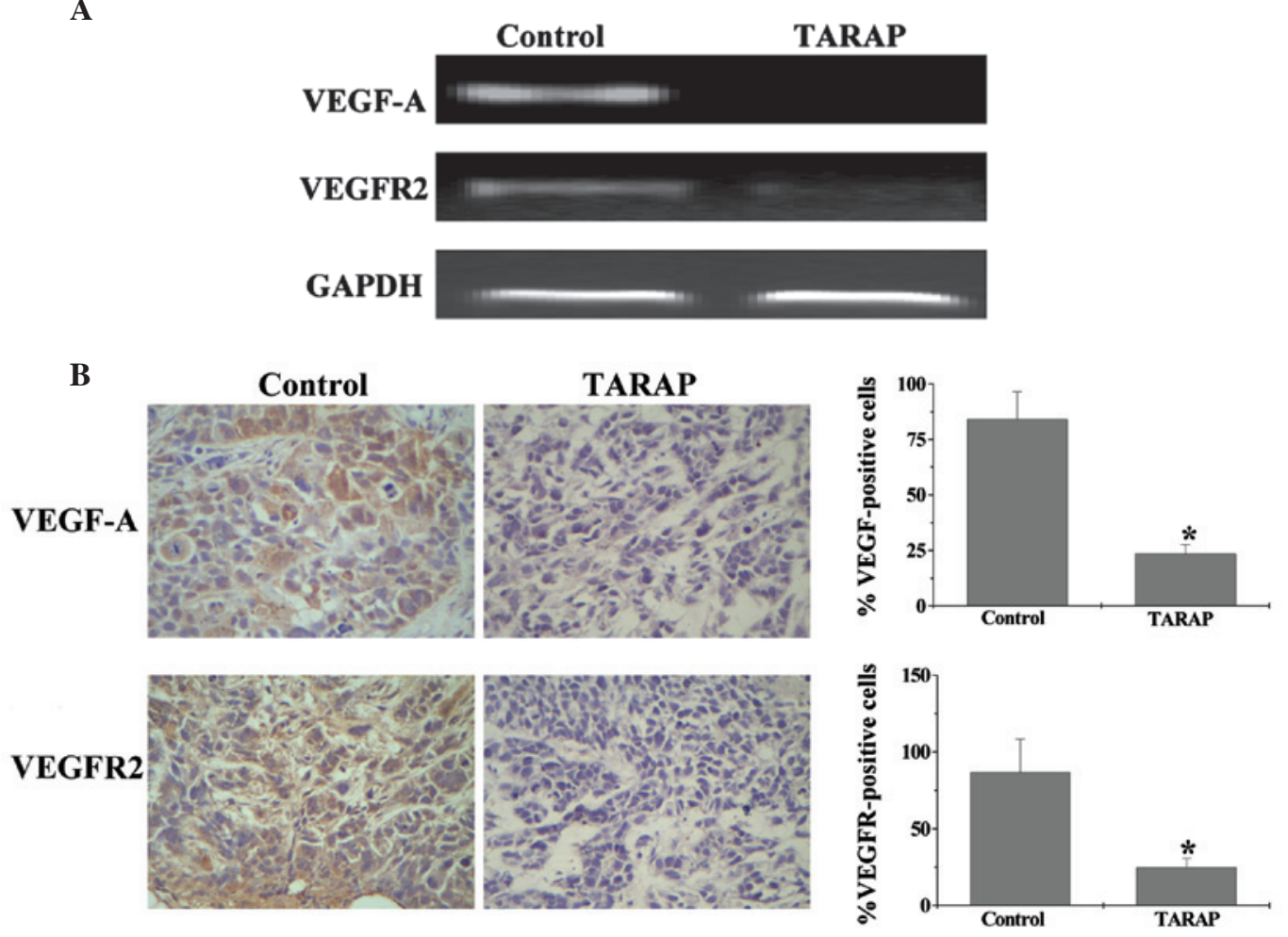

Figure 2. Effect of TARAP on the expression of VEGF-A and VEGFR2 in HCC xenograft mice. (A) mRNA expression of VEGF-A and VEGFR2 was determined using reverse transcription polymerase chain reaction analysis. GAPDH was used as the internal control. (B) Tumor tissues were processed for immunohistochemical staining for VEGF-A and VEGFR2. Images were captured at a magnification of x400. Immunohistochemical staining was quantified and represented as the percentage of positively-stained cells. Data are presented as the mean \pm standard deviation from 10 individual mice in each group. " $\mathrm{P}<0.05$ vs. control group. TARAP, total alkaloids of Rubus alceifolius Poir; HCC, hepatocellular carcinoma; VEGFR, vascular endothelial growth factor receptor.

hematoxylin. Subsequent to staining, five high-power fields (magnification, $\mathrm{x} 400$ ) were randomly selected in each slide and the average proportion of positive cells in each field was counted using the true color multi-functional cell image analysis management system (Image-Pro Plus; Media Cybernetics, Inc., Bethesda, MD, USA). To exclude any non-specific staining, PBS was used to replace the primary antibody as a negative control.

Statistical analysis. All data are presented as the mean \pm standard deviation. Data were analyzed using the SPSS package for Windows (Version 11.5; SPSS, Inc., Chicago, IL, USA). Statistical analysis of the data was performed using the Student's t-test. $\mathrm{P}<0.05$ was considered to indicate a statistically significant difference.

\section{Results and Discussion}

TARAP inhibits tumor angiogenesis in a xenograft model. Deregulated angiogenesis has a crucial role in the development of various diseases, including cancer $(6,22,23)$. CD31 is a characteristic molecule in vascular ECs which is used as an angiogenesis marker in vivo. In the present study, immunohistochemical staining of CD31 was used to identify the degree of angiogenesis. As shown in Fig. 1A and B, the microvessel 
A

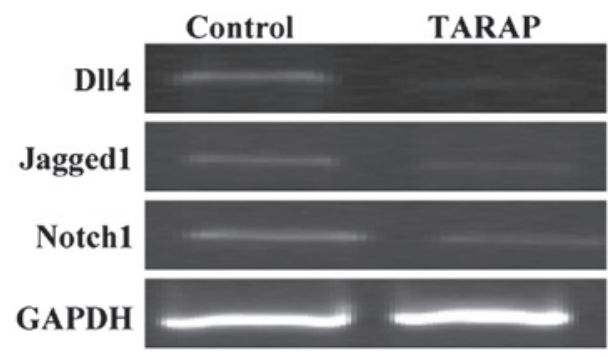

Ba

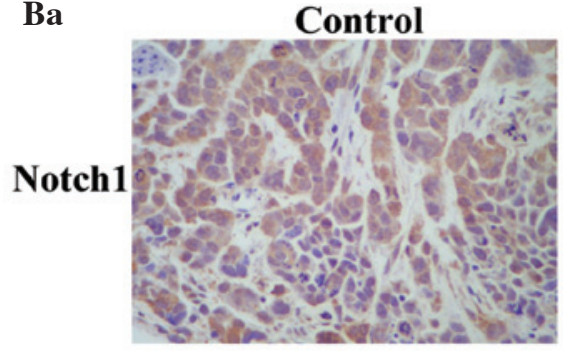

$\mathbf{B b}$

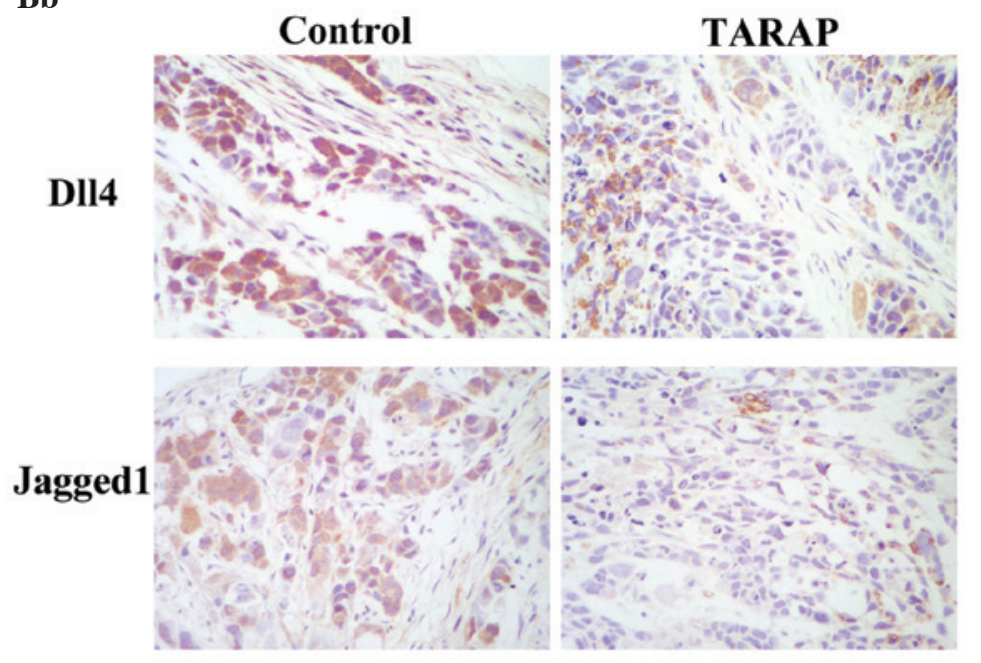

TARAP
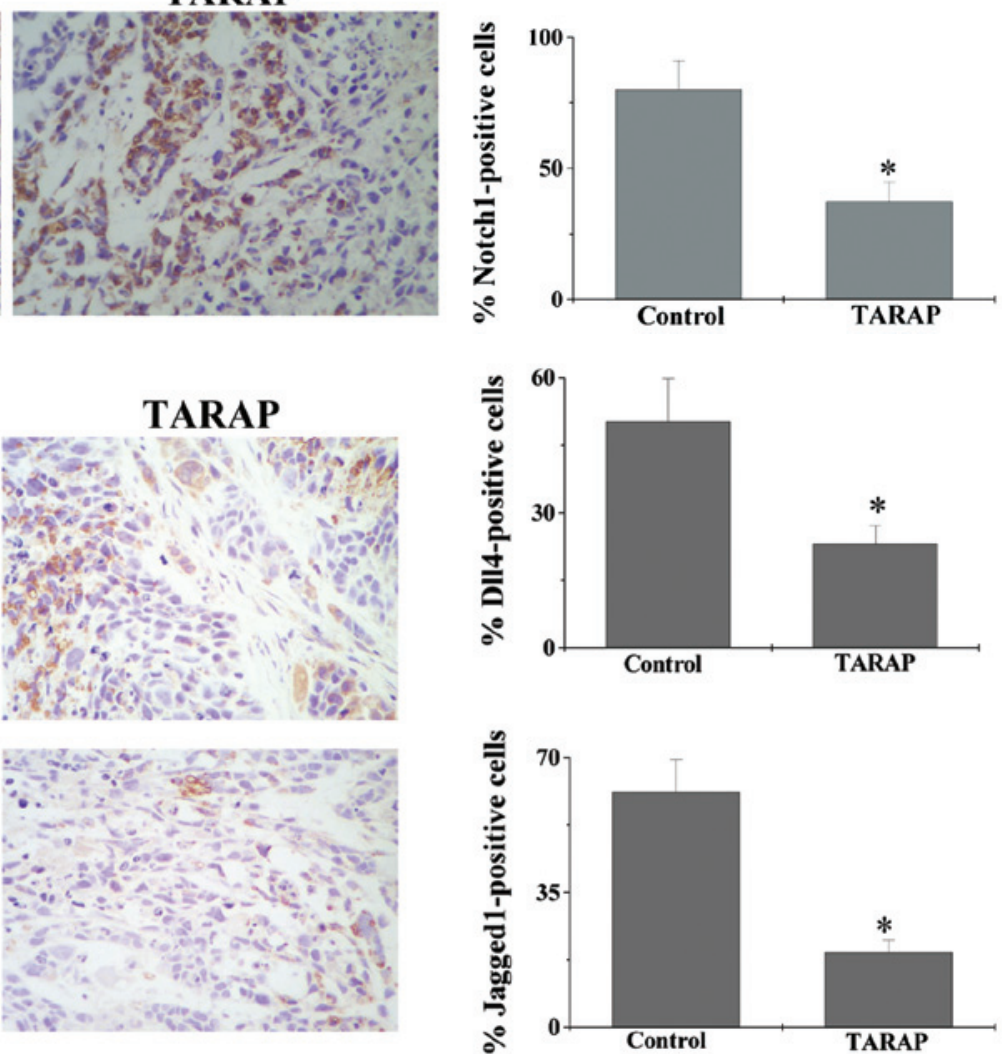

Figure 3. Effect of TARAP on the activation of the Notch signaling pathway in HCC xenograft mice. (A) mRNA expression of Notch1, jagged 1 and Dll4 was determined using polymerase chain reaction analysis. GAPDH was used as the internal control. (B) Tumor tissues were processed for immunohistochemical staining for $(\mathrm{Ba})$ Notch1 and (Bb) jagged 1 and Dll4. Images were captured at a magnification of $\mathrm{x} 400$. Immunohistochemical staining was quantified and presented as the percentage of positively stained cells. Data are presented as the mean \pm standard deviation from 10 individual mice in each group. ${ }^{*} \mathrm{P}<0.05$ vs. control group. TARAP, total alkaloids of Rubus alceifolius Poir; HCC, hepatocellular carcinoma; Dll, delta-like ligand.

density of the HCC xenografts in the TARAP-treated mice was observed to be reduced compared with that in the control mice, suggesting that TARAP inhibited angiogenesis in vivo. Thus, TARAP may mediate its antitumor activity through blocking angiogenesis.

TARAP inhibits the expression of VEGF-A and VEGFR2 in $H C C$ xenograft mice. In the process of tumor growth, interaction between tumor cells and vascular ECs causes increases in tumor blood vessels and promotes tumor growth $(24,25)$. VEGF-A is commonly expressed in a wide variety of types of human tumor cells, is one of the most effective biological inducers of angiogenesis and is associated with angiogenesis, growth and metastasis (26-29). To analyze the effect of TARAP on tumor angiogensis, VEGF-A and VEGFR2 expression were assessed in a xenograft model using PCR analysis and IHC. PCR analysis revealed that TARAP treatment reduced the mRNA expression of VEGF-A and VEGFR2 in the HCC xenografts (Fig. 2A). Furthermore, IHC showed that the protein expression of VEGF-A and VEGFR2 was similar to their respective mRNA expression levels. The percentage of VEGF-A- and VEGFR2-positive cells in the control group was found to be $84 \pm 12.34$ and $86.67 \pm 11.48 \%$, respectively, while that in TARAP-treated mice was $23.5 \pm 4.31$ and $24.83 \pm 5.79 \%$, respectively (Fig. 2B).

TARAP suppresses the Notch signaling pathway in HCC xenograft mice. Tumor angiogenesis is tightly regulated by the Notch signaling pathway; therefore, in the present study, the effect of TARAP on the expression of key mediators of the Notch signaling pathway was investigated using PCR and IHC analyses. As shown in Fig. 3A, TARAP treatment was observed to significantly reduce the mRNA expression of Notch, Dll4 and jagged 1 in tumor tissues. Furthermore, 
their protein expression was significantly inhibited by TARAP treatment. The percentages of Notch-, Dll4- and jagged 1-positive cells in the control group were $80 \pm 10.96,50.17 \pm 9.68$ and $61 \pm 8.42 \%$, respectively, whereas those in TARAP-treated mice were $37.33 \pm 7.32,23.17 \pm 3.99$ and $19.5 \pm 3.1 \%$, respectively (Fig. 3B). These findings suggested that the inhibitory effect of TARAP on tumor angiogenesis in vivo may be mediated through the suppression of the Notch signaling pathway.

In conclusion, to the best of our knowledge, the present study was the first study to show that TARAP inhibits tumor angiogenesis through the suppression of the Notch signaling pathway, which may in part explain its anticancer activity.

\section{Acknowledgements}

The present study was supported by grants from the Nature Science Foundation of Fujian Province of China (nos. 2010J01191 and 2010J01194) and the Medical Originality Foundation of Fujian Province of China (no. 2009-CX-18).

\section{References}

1. Tsai WL and Chung RT: Viral hepatocarcinogenesis. Oncogene 29: 2309-2324, 2010.

2. Schütte K, Bornschein J and Malfertheiner P: Hepatocellular carcinoma - epidemiological trends and risk factors. Dig Dis 27: 80-92, 2009.

3. Bikfalvi A: Significance of angiogenesis in tumour progression and metastasis. Eur J Cancer 31A: 1101-1104, 1995.

4. Folkman J: What is the evidence that tumors are angiogenesis dependent? J Natl Cancer Inst 82: 4-6, 1990.

5. Ingber D, Fujita T, Kishimoto S, Sudo K, Kanamaru T, Brem H and Folkman J: Synthetic analogues of fumagillin that inhibit angiogenesis and suppress tumour growth. Nature 348: 555-557, 1990.

6. Folkman J and Shing Y: Angiogenesis. J Biol Chem 267: 10931-10934, 1992.

7. Pluda JM: Tumor-associated angiogenesis: mechanisms, clinical implications, and therapeutic strategies. Semin Oncol 24: 203-218, 1997.

8. Folkman J: Anti-angiogenesis: new concept for therapy of solid tumors. Ann Surg 175: 409-416, 1972.

9. Brem $\mathrm{H}$ and Folkman J: Inhibition of tumor angiogenesis mediated by cartilage. J Exp Med 141: 427-439, 1975.

10. Voest EE: Inhibitors of angiogenesis in a clinical perspective. Anticancer Drugs 7: 723-727, 1996.

11. Jung YD, Ahmad SA, Liu W, Reinmuth N, Parikh A, Stoeltzing O, Fan F and Ellis LM: The role of the microenvironment and intercellular cross-talk in tumor angiogenesis. Semin Cancer Biol 12: $105-112,2002$.
12. Krebs LT, Xue Y, Norton CR, et al: Notch signaling is essential for vascular morphogenesis in mice. Genes Dev 14: 1343-1352, 2000.

13. Krebs LT, Shutter JR, Tanigaki K, Honio T, Stark KL and Gridley T: Haploinsufficient lethality and formation of arteriovenous malformations in Notch pathway mutants. Genes Dev 18 : 2469-2473, 2004.

14. Xue Y, Gao X, Lindsell CE, et al: Embryonic lethality and vascular defects in mice lacking the Notch ligand Jagged1. Hum Mol Genet 8: 723-730, 1999.

15. Artavanis-Tsakonas S, Rand MD and Lake RJ: Notch signaling: cell fate control and signal integration in development. Science 284: 770-776, 1999.

16. Guo S, Liu M and Gonzalez-Perez RR: Role of Notch and its oncogenic signaling crosstalk in breast cancer. Biochim Biophys Acta 1815: 197-213, 2011.

17. Li JL and Harris AL: Notch signaling from tumor cells: a new mechanism of angiogenesis. Cancer Cell 8: 1-3, 2005.

18. Taylor KL, Henderson AM and Hughes CC: Notch activation during endothelial cell network formation in vitro targets the basic HLH transcription factor HESR-1 and downregulates VEGFR-2/KDR expression. Microvasc Res 64: 372-383, 2002.

19. Williams CK, Li JL, Murga M, Harris AL and Tosato G: Upregulation of the Notch ligand Delta-like 4 inhibits VEGF-induced endothelial cell function. Blood 107: 931-939, 2006.

20. Zimrin AB, Pepper MS, McMahon GA, Nguyen F, Montesano R and Maciag T: An antisense oligonucleotide to the notch ligand jagged enhances fibroblast growth factor-induced angiogenesis in vitro. J Biol Chem 271: 32499-32502, 1996.

21. Zhao J, Chen X, Lin W, et al: Total alkaloids of Rubus aleaefolius Poir inhibit hepatocellular carcinoma growth in vivo and in vitro via activation of mitochondrial-dependent apoptosis. Int J Oncol 42: 971-978, 2013.

22. Folkman J: Angiogenesis in cancer, vascular, rheumatoid and other diseases. Nat Med 1: 27-31, 1995.

23. Folkman J: Angiogenesis. Annu Rev Med 57: 1-18, 2006.

24. Yancopoulos GD, Davis S, Gale NW, et al: Vascular-specific growth factors and blood vessel formation. Nature 407: 242-248, 2000 .

25. Ishiwata I, Sudo T, Kiguchi K and Ishikawa H: Tumor angiogenesis factors produced by cancer cells. Hum Cell 12: 37-46, 1999.

26. Risau W: Mechanisms of angiogenesis. Nature 386: 671-674, 1997.

27. Jain RK: Tumor angiogenesis and accessibility: role of vascular endothelial growth factor. Semin Oncol 29 (6 Suppl 16): 3-9, 2002.

28. Ferrara N: Role of vascular endothelial growth factor in physiologic and pathologic angiogenesis: therapeutic implications. Semin Oncol 29 (6 Suppl 16): 10-14, 2002.

29. Houck KA, Ferrara N, Winer J, et al: The vascular endothelial growth factor family: identification of a fourth molecular species and characterization of alternative splicing of RNA. Mol Endocrinol 5: 1806-1814, 1991. 